\title{
THE POSTDOC VARIANT OF THE SECRETARY PROBLEM
}

\author{
ROBERT J. VANDERBEI
}

\begin{abstract}
The classical secretary problem involves sequentially interviewing a pool of $n$ applicants with the aim of hiring exactly the best one in the pool—nothing less is good enough. The optimal decision strategy is easy to describe and the probability of success is $1 / e$. In this paper, we consider a minor variant of this classical problem. We wish to pick not the best but the second best (the best is going to Harvard). In this case, an explicit solution can be given both for the optimal strategy and the associated optimal success probability. The probability of success is $k_{0}\left(n-k_{0}\right) /(n(n-1))$ where $k_{0}=\lfloor n / 2\rfloor$. Clearly, as $n$ goes to infinity, the probability of success tends to $1 / 4$. Apparently, it is easier to pick the best than the second best.
\end{abstract}

\section{INTRODUCTION.}

The secretary problem (first popularized by Martin Gardner [4]) is a classical problem in optimal selection. Dynkin [2] and, in slightly different form, Chow et al. [1] were the first to give rigorous treatments. The problem is described as follows. A manager wishes to hire a secretary. An ad is posted and $n$ applicants apply. The candidates are interviewed in random order-nothing is known about their qualifications prior to showing up for the interview. At the conclusion of the interview, the manager must decide either to hire this applicant or not. The decision cannot be postponed until the manager has seen more applicants. The decision regarding each candidate must be made as the candidate is being interviewed. There are no second chances. If the manager exhausts all candidates and doesn't hire anyone, not even the last applicant, then the process is considered a failure. Furthermore, if the manager hires someone but that person turns out not to be the absolute best of all $n$ candidates, then that, too, will be considered a failure. The only successful outcome is to hire one candidate and that one must be the best of the bunch. All other outcomes are considered equally bad-failures. This is the secretary problem.

The problem became famous when it was first proposed because it turns out that the manager's optimal strategy is truly elegant. Here's the optimal strategy. The manager should interview the first $n / e$ applicants (where $e=2.71828 \ldots$ ) and reject them categorically. In modern terms, this is called the exploration part of the process. The manager is simply trying to calibrate the quality of the pool of applicants. Obviously, the manager hopes that the best candidate is not among this

Date: November 6, 2011.

Key words and phrases. Secretary problem, online learning, online auctions. 
initial pool for, in that case, the hiring process will be a failure. Anyway, after rejecting the first $n / e$ applicants, the manager will hire the first subsequent applicant who is superior to all who have come before. Of course, even in this decision, there is the risk that the best is yet to come and so the process could still end in failure. Nonetheless, this is the optimal strategy. It is well-known that the probability of success is approximately $1 / e$ and converges to this value as $n$ goes to infinity.

Over the years, many variants of the secretary problem have been formulated and solved. GuseinZade [5] studied the problem of picking one of the $k$ best out of $n$ and Vanderbei [7] considered the problem of picking all of the $k$ best out of $n$. Freeman [3] provides a review of the many variants that were developed by that time.

\section{The Postdoc Problem.}

In 1980, E.B. Dynkin proposed (to the author) the problem of picking exactly the second best candidate. The motivating story was adapted to this problem: we are trying to hire a postdoc and we are confident that the best applicant will receive and accept an offer from Harvard.

We model this as a sequential (online) decision problem. For $k=0,1, \ldots, n$, let $v_{k}$ denote the probability of success using the optimal strategy assuming that $k$ candidates have already been interviewed and so far none of them have been hired. Our goal is to write and then solve the Hamilton-Jacobi-Bellman (HJB) equation for $v$. In order to write the HJB equation, we need two precomputed sequences.

We assume that we interview the candidates one at a time and make our decision on the spot. But, even after a decision is made, we continue to interview the remaining candidates simply to see if we indeed made the correct choice. Suppose after interviewing $k$ candidates, one of those $k$ has been selected and the selected candidate is second best among the $k$ seen so far. Let $g_{k}$ denote the probability that this currently-second-best candidate will still be second-best after we have seen all $n$ candidates. It is easy to give a formula for $g_{k}$. Consider what can happen when the $(k+1)$-st applicant is interviewed. It could be the new best, or the new second best, or etc., or the new last best (i.e., worst). There are $k+1$ relative positions it could acquire. Since we have no a priori knowledge about this (or any) candidate, he/she is equally likely to fall into any of these $k+1$ slots in the ranking. Hence, with probability $2 /(k+1)$, this new applicant will be either first or second best among the $k+1$ seen so far. In this case, the applicant who has already been hired will drop to third place and the hiring process will have been a failure. On the other hand, with probability $(k-1) /(k+1)$, this newly interviewed applicant will be worse than the one we've actually hired and so the hired candidate will remain in second place. Hence,

$$
g_{k}=\left\{\begin{array}{lr}
\frac{k-1}{k+1} g_{k+1}, & 2 \leq k<n, \\
1, & k=n .
\end{array}\right.
$$


This equation is trivial to solve:

$$
\begin{aligned}
g_{k} & =\frac{k-1}{k+1} \frac{k}{k+2} \frac{k+1}{k+3} \cdots \frac{n-3}{n-1} \frac{n-2}{n} g_{n} \\
& =\frac{k(k-1)}{n(n-1)} .
\end{aligned}
$$

Now, suppose that after interviewing $k$ applicants, one has been hired and this one is currently the best of those seen so far (we are hoping there is still one better applicant among those not yet interviewed). Let $f_{k}$ denote the probability that the hired applicant will be ranked second best after seeing all applicants. As before, consider the next candidate. He/she is going to be either the best so far, in which case the hired candidate will drop to second place, or not, in which case the hired candidate remains best among those seen so far. Hence, we can write

$$
f_{k}=\left\{\begin{array}{lr}
\frac{k}{k+1} f_{k+1}+\frac{1}{k+1} g_{k+1}, & 1 \leq k<n, \\
0, & k=n .
\end{array}\right.
$$

This equation is also easy to solve. Let $h_{k}=f_{k} / k$. Then, using the explicit formula for $g_{k}$, the equation for $h_{k}$ is especially simple:

$$
h_{k}=h_{k+1}+\frac{1}{n(n-1)} .
$$

Hence, using $h_{n}=0$, we get

$$
h_{k}=\frac{n-k}{n(n-1)}
$$

and therefore

$$
f_{k}=\frac{k(n-k)}{n(n-1)}, \quad 1 \leq k \leq n
$$

\section{The Value Function}

Now suppose that we have interviewed $k$ applicants and have rejected all of them. The value function $v_{k}, k=0,1, \ldots, n$, is defined as the probability, using the best possible strategy, of eventually hiring someone who turns out to be the second-best candidate.

According to the principle of dynamic programming, it suffices to do the right thing at each stage. So, suppose that $k$ applicants have been interviewed and no one has been hired so far. Consider the $(k+1)$-st candidate. With probability $(k-1) /(k+1)$ this new interviewee will be worse that the best and second-best seen so far. Hence, there is no reason to hire this candidate. In this case, we proceed to stage $k+1$ also with no one hired. 
Suppose, however, that the $(k+1)$-st candidate is actually better than all $k$ seen so far. Now we have a choice: we could hire this candidate and hope that he/she drops to second-best after all have been seen or we could pass on this candidate and proceed to the $(k+1)$-st stage without making a hire. If we hire, the probability of success is $f_{k+1}$. If we pass, the probability of success is $v_{k+1}$. We should pick the larger.

Finally, suppose that the $(k+1)$-st candidate is second best among all seen so far. Again, we have a choice: hire this candidate and hope that he/she remains second-best until the end or pass on this candidate and proceed to the $(k+1)$-st stage without making a hire. If we hire, the probability of success is $g_{k+1}$. If we pass, the probability of success is $v_{k+1}$.

Putting these possibilities together, we get the following Hamilton-Jacobi-Bellman (HJB) equation for $v_{k}$ :

$$
v_{k}=\left\{\begin{array}{lr}
\max \left(v_{k+1}, f_{k+1}\right), & k=0, \\
\frac{k-1}{k+1} v_{k+1}+\frac{1}{k+1} \max \left(v_{k+1}, f_{k+1}\right)+\frac{1}{k+1} \max \left(v_{k+1}, g_{k+1}\right), & 1 \leq k<n, \\
0, & k=n .
\end{array}\right.
$$

Let $k_{0}=\min \{k \mid 2 k \geq n-1\}$. It is easy to check that $k_{0}=n / 2$ if $n$ is even and $k_{0}=(n-1) / 2$ if $n$ is odd. The following theorem gives the specific formula for $v_{k}$.

Theorem 1. The value function is given by

$$
v_{k}= \begin{cases}\frac{k(n-k)}{n(n-1)}, & k_{0} \leq k \leq n, \\ \frac{k_{0}\left(n-k_{0}\right)}{n(n-1)}, & 0 \leq k<k_{0} .\end{cases}
$$

Proof. Clearly (3) is correct for $k=n$ as the formula reduces to $v_{n}=0$. Suppose that the formula is correct for a fixed $k>k_{0}$. We shall prove that it is also correct for $k-1$. First note that, 
according to the induction hypothesis, $v_{k}=f_{k}$. Also, $v_{k} \leq g_{k}$ since $k \geq k_{0}$. Hence

$$
\begin{aligned}
v_{k-1} & =\frac{k-2}{k} v_{k}+\frac{1}{k} \max \left(v_{k}, f_{k}\right)+\frac{1}{k} \max \left(v_{k}, g_{k}\right) \\
& =\frac{k-2}{k} v_{k}+\frac{1}{k} v_{k}+\frac{1}{k} g_{k} \\
& =\frac{(k-1)(n-k)}{n(n-1)}+\frac{(k-1)}{n(n-1)} \\
& =\frac{(k-1)(n-(k-1))}{n(n-1)}
\end{aligned}
$$

Now, suppose that (3) is correct for a specific $2 \leq k \leq k_{0}$. By induction, we need to check it for $k-1$. In this case, we claim that $v_{k} \geq f_{k}$ and that $v_{k} \geq g_{k}$ and hence

$$
\begin{aligned}
v_{k-1} & =\frac{k-2}{k} v_{k}+\frac{1}{k} \max \left(v_{k}, f_{k}\right)+\frac{1}{k} \max \left(v_{k}, g_{k}\right) \\
& =\frac{k-2}{k} v_{k}+\frac{1}{k} v_{k}+\frac{1}{k} v_{k} \\
& =v_{k} \\
& =\frac{k_{0}\left(n-k_{0}\right)}{n(n-1)}
\end{aligned}
$$

Hence, it suffices to check the two claimed inequalities.

The first claim is that $v_{k} \geq f_{k}$. This inequality is equivalent to

$$
k_{0}\left(n-k_{0}\right) \geq k(n-k) .
$$

Let $p$ denote the polynomial given by $p(x)=x(n-x)$. This quadratic has its maximum at $x=n / 2$. Since, $k \leq k_{0} \leq n / 2$, it follows that $p(k) \leq p\left(k_{0}\right)$, which is the first claim.

The second claim is that $v_{k} \geq g_{k}$, which is equivalent to

$$
k_{0}\left(n-n_{0}\right) \geq k(k-1) .
$$

We already have shown that $k_{0}\left(n-k_{0}\right) \geq k(n-k)$. Hence, it suffices to show that

$$
k(n-k) \geq k(k-1) \text {. }
$$

For $k \neq 0$, this inequality is equivalent to $k \leq(n+1) / 2$ and hence it holds for any $k \leq k_{0}$.

Finally, we must check $v_{0}$. Since, $v_{1} \geq f_{1}$, it follows that $v_{0}=v_{1}$.

The value function determines not only the probability of success, $v_{0}$, but also the optimal strategy that achieves this probability. Specifically, the strategy is determined by noting which number achieves the maximum in the last two terms of the HJB equation. 
Theorem 2. The value function $v_{k}, 0 \leq k \leq n$, satisfies the following equalities and inequalities:

$$
\begin{array}{ll}
v_{k}>f_{k}, & \text { for } k<k_{0}, \\
v_{k}=f_{k}, & \text { else }
\end{array}
$$

and, for $n$ even,

$$
\begin{aligned}
& v_{k}>g_{k}, \quad \text { for } k \leq k_{0}, \\
& v_{k}<g_{k}, \quad \text { else, }
\end{aligned}
$$

whereas, for $n$ odd,

$$
\begin{array}{ll}
v_{k}>g_{k}, & \text { for } k \leq k_{0}, \\
v_{k}=g_{k}, & \text { for } k=k_{0}+1, \\
v_{k}<g_{k}, & \text { else. }
\end{array}
$$

Proof. The proof follows trivially from the previous Theorem.

The previous theorem determines the optimal strategy. Because of the various equalities, there are many situations of indifference. For example, for $k \geq k_{0}$, we can either accept or reject a candidate that if best-so-far. We end by stating one optimal strategy that is easy to remember:

Reject the first $k_{0}$ applicants. After that hire the first second-best-so-far applicant that comes along.

Using such an optimal strategy, the probability of successfully hiring the second-best applicant is

$$
v_{0}=\frac{k_{0}\left(n-k_{0}\right)}{n(n-1)} \approx 1 / 4
$$

Figure 1 shows a plot of $f_{k}, g_{k}$, and $v_{k}$ for an even value of $n$ while Figure 2 shows a similar plot for an odd value.

\section{DigGing DeEPER.}

Suppose, instead of the second best, we wish to select the $m$-th best out of $n$. This problem can be attacked in a manner analogous to that of the previous section. Indeed, let $f_{j, k}$ denote the probability of ending up with the $m$-th best choice given that this person is currently known to be 


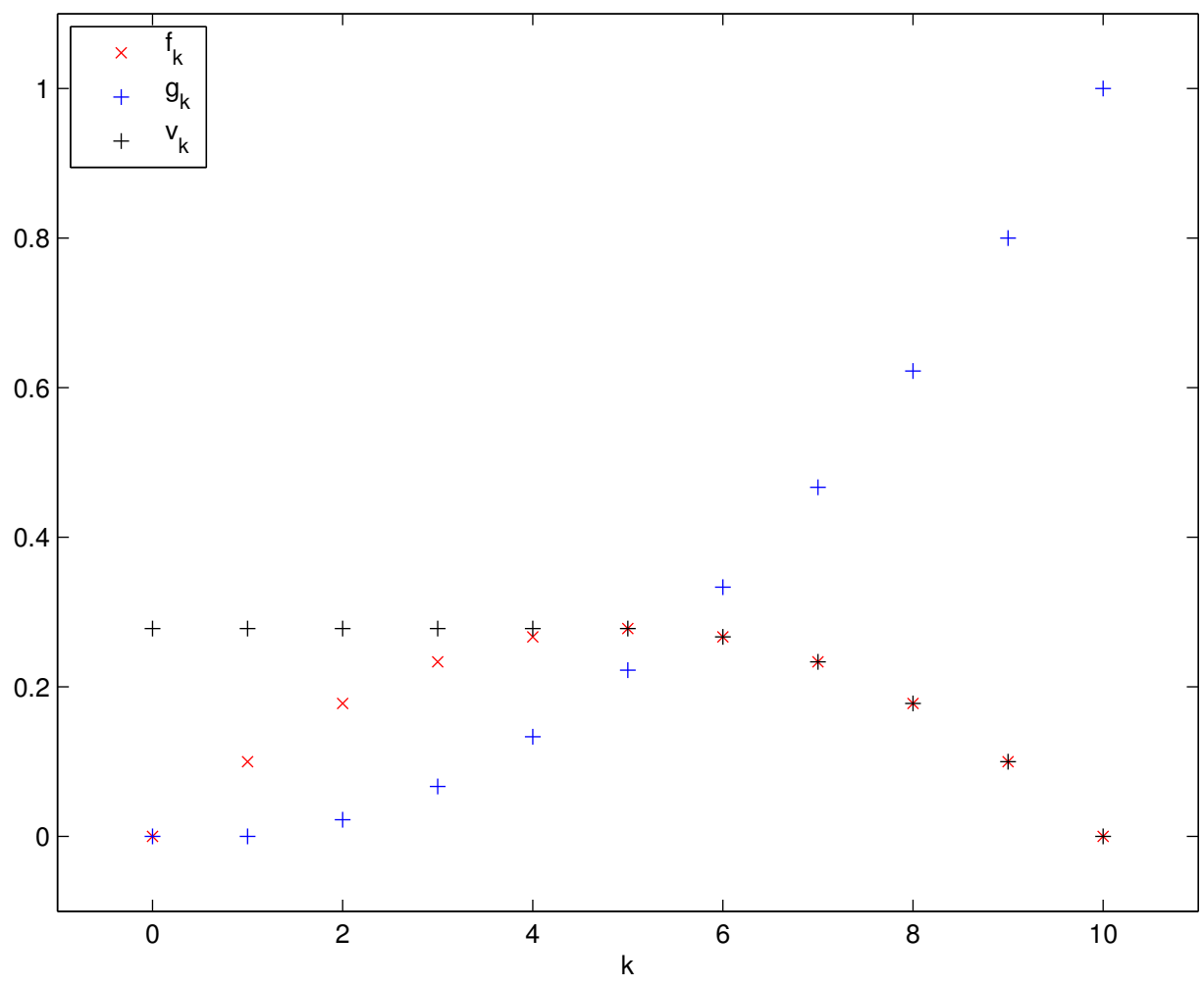

FIgURE 1. A plot of $f_{k}, g_{k}$, and $v_{k}$ for $n=10$.

the $j$-th best of out the first $k$ interviewed. It is easy see that

$$
f_{j, k}=\left\{\begin{array}{lr}
\frac{k-j+1}{k+1} f_{j, k+1}+\frac{j}{k+1} f_{j+1, k+1}, & j \leq k<n, \\
1, & j=m, k=n, \\
0, & j \neq m, k=n .
\end{array}\right.
$$

Clearly, $f_{m+1, k}=0$ for all $k$.

Theorem 3. For $j \leq m$,

$$
f_{j, k}=\frac{\left(\begin{array}{c}
k \\
j
\end{array}\right)\left(\begin{array}{c}
n-k \\
m-j
\end{array}\right)}{\left(\begin{array}{c}
n \\
m
\end{array}\right)} \frac{j}{m}
$$




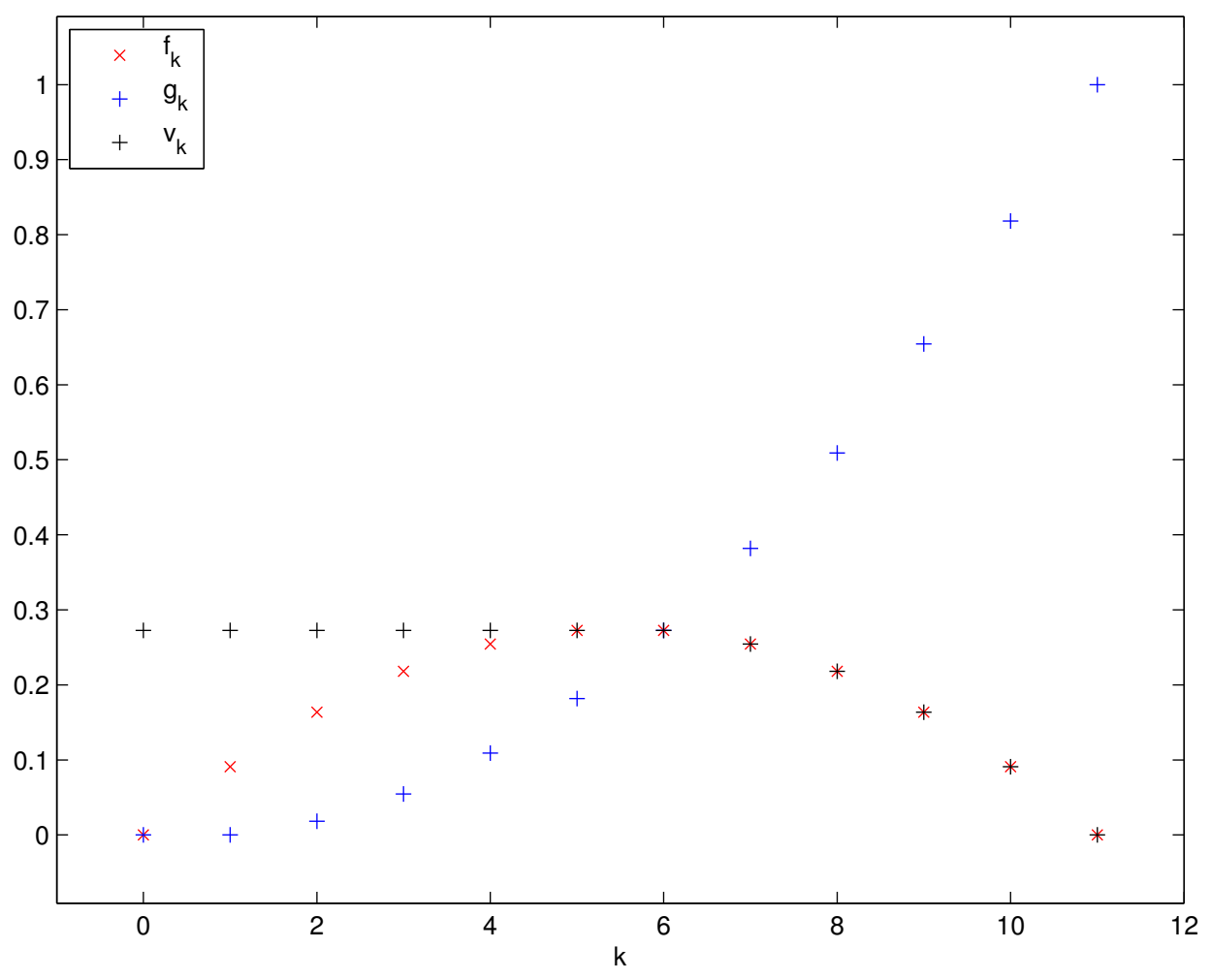

FIGURE 2. A plot of $f_{k}, g_{k}$, and $v_{k}$ for $n=11$.

Proof. Backward induction on $j$ and $k$ proves that $f_{j, k}$ is unique. Hence, it suffices to check that the formula given actually satisfies the defining equation. We compute

$$
\begin{aligned}
\frac{k-j+1}{k+1} f_{j, k+1} & +\frac{j}{k+1} f_{j+1, k+1} \\
& =\frac{k-j+1}{k+1} \frac{\left(\begin{array}{c}
k+1 \\
j
\end{array}\right)\left(\begin{array}{c}
n-k-1 \\
m-j
\end{array}\right)}{\left(\begin{array}{c}
n \\
m
\end{array}\right)} \frac{j}{m}+\frac{j}{k+1} \frac{\left(\begin{array}{c}
k+1 \\
j+1
\end{array}\right)\left(\begin{array}{c}
n-k-1 \\
m-j-1
\end{array}\right)}{\left(\begin{array}{c}
n \\
m
\end{array}\right)} \frac{j+1}{m} \\
& =\frac{\left(\begin{array}{c}
k \\
j
\end{array}\right)\left(\begin{array}{c}
n-k-1 \\
m-j
\end{array}\right)}{\left(\begin{array}{c}
n \\
m
\end{array}\right)} \frac{j}{m}+\frac{\left(\begin{array}{c}
k \\
j
\end{array}\right)\left(\begin{array}{c}
n-k-1 \\
m-j-1
\end{array}\right)}{\left(\begin{array}{c}
n \\
m
\end{array}\right)} \frac{j}{m} \\
& =\frac{\left(\begin{array}{c}
k \\
j
\end{array}\right)\left(\begin{array}{c}
n-k \\
m-j
\end{array}\right)}{\left(\begin{array}{c}
n \\
m
\end{array}\right)} \frac{j}{m} \\
& =f_{j, k} .
\end{aligned}
$$


As before, let $v_{k}$ denote the optimal probability of success given that $k$ candidates have been interviewed and rejected. The HJB equation for $v_{k}$ is

$$
v_{k}=\frac{k-m+1}{k+1} v_{k+1}+\sum_{j=1}^{m} \frac{1}{k+1} \max \left(v_{k+1}, f_{j, k+1}\right) .
$$

Unfortunately, except for the cases $m=1$ (the traditional secretary problem), $m=2$ (the pick the second-best problem), and, by symmetry, $m=n$ and $m=n-1$, no other cases have explicit solutions.

They are, however, easy to compute numerically. The problem of picking the median candidate from an odd-sized pool seems particularly interesting.

\section{CONCLUSiOns.}

We have given an explicit solution to the problem of finding the second-best from a pool of $n$ "postdoc" applicants. It turns out that this problem has a more explicit solution than the traditional secretary problem. Specifically, the optimal strategy is to reject the first half of the applicants and then to accept the first second-best-so-far applicant that arrives after rejecting the first half. The probability of success using this strategy is about 1/4. Apparently, it is harder to select the second-best from a pool of applicants than it is to select the best.

Of course, the result in this paper raises the question: what about picking the 3rd best, or the 4th best, or, more generally, the $k$-th best. We leave these investigations to future work.

Acknowledgements. The author would like to thank Eugene Dynkin for suggesting this problem to him thirty-two years ago (the problem was solved in a few days but only written up for publication now). The author would also like to thank XXX for reading a draft of this paper and providing numerous helpful suggestions.

The author recently learned that the second-best problem was solved by John Rose [6].

\section{REFERENCES}

[1] Y.S. Chow, S. Moriguti, H. Robbins, and S.M. Samuels. Optimal selection based on relative rank. Israel J. Math., 2:81-90, 1964.

[2] E.B. Dynkin. The optimum choice of the instant for stopping a Markov process. Dokl. Akad. Nauk SSSR, 150:238$240,1963$. 
[3] P.R. Freeman. The secretary problem and its extensions-a review. Intl. Statistical Review, 51:189-206, 1983.

[4] M. Gardner. Mathematical games, 1960.

[5] S.M. Gusein-Zade. The problem of choice and the optimal stopping rule for a sequence of independent trials. Theory Prob. and its Appl., 11:472-476, 1966.

[6] J.S. Rose. A problem of optimal choice and assignment. Operations Research, 30:172-181, 1982.

[7] R.J. Vanderbei. Optimal choice of a subset of a population. Math. OR, 5:481-486, 1980.

Department of Operations Research and Financial Engineering, Princeton University, PrinceTON, NJ 08544.

E-mail address: rvdbeprinceton . edu 